\title{
Study of Thermodynamic Properties of Monoclinic Sulfur (Sß) Under High Pressure Using Three Different Equations of State for the Treatment Scabies in Dermatology
}

\author{
Abdullah M. Ali ${ }^{1}$, Raed Hashim AL-Saqa ${ }^{2 *}$, Nashwa Salhuddin Sultan ${ }^{3}$ \\ ${ }^{1}$ Department of physics, University of Tikrit / Iraq \\ ${ }^{2}$ Directorate General of Education/Nineveh / Iraq \\ ${ }^{3}$ Almutamayizat High School, Directorate General of Education/ Iraq \\ E-mail: *raed1970ah@yahoo.com
}

Received 3 October 2021, Revised 6 December 2021, Accepted 24 January 2022

\begin{abstract}
Thermodynamic properties of monoclinic $\beta$-sulfur $S_{\beta}$ under high pressure up to 20 Gpa have been studied, where Orthorhombic $\alpha$-sulfur $S_{\alpha}$ changes to $S_{\beta}$ at $94.4^{\circ} \mathrm{C}$. The high pressure technology used to tackle the crystallization volume of sulfur $S_{\beta}$ without changes in the chemical properties. Sulfur $S_{\beta}$ can be used after the effect of high pressure in the development of the medicine used to treat scabies. The present study is performed to calculate the effects of high pressure on some important physical properties of the material such as (bulk modulus (B), compression volume ratio $\left(\mathrm{V}_{\mathrm{P}} / \mathrm{V}_{\mathrm{o}}\right)$, Grüneisen parameter $(\gamma)$, Debye temperature $\left(\theta_{\mathrm{D}}\right)$ and phonon frequency spectrum (pfs). Three different equations of state EOS's (Birch- Murnaghan EOS, Vinet EOS and modified Lennard-Jones EOS) were implemented to analyze pressure-volume relationship and then combining calculated data with various expressions of volume dependence of the other thermodynamic properties that are; Bulk modulus, Grüneisen parameter, Debye temperature and phonon frequency spectrum. The behavior of any of these concepts were described in different figures. It was found that, relative volume, and Grüneisen parameter were decreased with high pressure, while the other considered parameters; Bulk modulus, Debye temperature and phonon frequency spectrum were expected to increase as a high pressure is applied.
\end{abstract}

Keywords: Bulk modulus; volume compression ratio; lattice parameter; phonon frequency spectrum; isothermal equations of state.

\section{Introduction}

In the present work, the bulk modulus (B), compression volume $\left(\mathrm{V}_{\mathrm{P}} / \mathrm{V}_{\mathrm{o}}\right)$, lattice constant (a) and phonon frequency spectrum (pfs) of a $S_{\beta}$ compound under the influence of high pressure were calculated using the "Birch-Murnaghan and modified Lennard-Jones" EOS. The calculated results have been compared with experimental data, which confirms the validity of the present equations of state. The Grüneisen parameter variation assumption has improved the results of phonon frequency spectrum under compression.

In this study, sulfur will be used to compress to make it smaller than its present in nature, where [1] observed that the materials in small size "Nano scale" can have more useful applications than their bulk counterpart.

Sulfur can be crystallized in some different lattices, the well-known orthorhombic structure of $S_{\alpha}$ comprising of $S_{8}$ rings [2], it is stable under high pressure up to (20-30) Gpa and became gradually amorphous on further compression [3, 4,5]. Recrystallization starts around $37 \mathrm{Gpa}$ and fully completes only at $75 \mathrm{Gpa}$ [6]. The structure of three solid allotropes as:

\subsection{Orthorhombic $\alpha$-Sulfur $S_{\alpha}$}

$\mathbf{S}_{\boldsymbol{\alpha}}$ is the stable form of Cycloocta-S. [7] has reported very accurate structure parameters. [8] established "the stacking of molecules", (Fig. 1a) show the "crankshaft" structure of this allotrope, is still erroneously assumed by many to contain coaxially stacked rings [7].

Lattice constants are " $\mathrm{a}=10.4633 \AA, \mathrm{b}=12.8786 \AA$, and $\mathrm{c}=24.4784 \AA$, and the density is $2.069 \mathrm{~g} / \mathrm{cm}^{3}$ "[9], where its unit cell volume $3299.5 \AA^{3}[10]$.

\subsection{Monoclinic $\boldsymbol{\beta}$ - Sulfur $\mathrm{S}_{\boldsymbol{\beta}}$}

$S_{\beta}$ forms at $94.4^{\circ} \mathrm{C}$ from $S_{\alpha}[11]$, its structure determined by Trillat and Forestler [12], the unite cell contain six molecules $\mathrm{S}_{8}$ i.e., 48 atoms, (Fig.1b), its lattice constant " $\mathrm{a}=10.778 \AA, \mathrm{b}=10.844 \AA$, and $\mathrm{c}=10.924 \AA$ " [13], and its density is $1.94 \mathrm{~g} / \mathrm{cm}^{3}$ [14], about $12 \%$ smaller than $S_{\alpha}$, and its unit cell volume $1276.41 \AA^{3}[15]$.

\section{$1.3 \gamma$-Monoclinic Sulfur $\mathbf{S}_{\gamma}$}

The structure has been determined by [16], who confirmed the "sheared penny roll" staking, proposed by De Haan [17] (Fig. 1c). "The lattice constants are: $\mathrm{a}=8.44 \AA$, $b=13.025 \AA, c=9.356 \AA$ " [16], four $\mathrm{S}_{8}$ molecules occupy one unite cell and its density $2.19 \mathrm{~g} / \mathrm{cm}^{3}$ (larger than $S_{\alpha}$ and $S_{\beta}$ sulfur) [16-17].

The purpose of studying (sulfur $S_{\beta}$ under high pressure), is for treating humans from some skin diseases specially (Scabies), where we note that its drug contains sulfur $5 \%$ for child and 10\% for young people [19-20]. 
In some cases, the treatment is time-consuming about (510) weeks or more. The reason, that sulfur is used in the treatment $S_{\alpha}$ type, which it has larger size than $S_{\beta}$ and therefore does not enter the pores of the skin [21]. using highpressure technology by three different equations of state to obtain a crystalline volume up to 0.65 of its original size after applying high pressure (about 20Gpa) to reach the skin pores size and grooves size that made by the Scabies germ. By comparing the dimensions of the pores with the grooves made by microscopic skin scabies under the skin, we will be able to make sulfur molecules interfere with skin pores and then into grooves created by bacteria and then kill them. In addition, the sulfur acts on the adhesion on the eggs completely so that it drowns grooves and thus kills the larvae that are born after (3-5) days.

The human body can be disinfected by treating sulfur $S_{\beta}$ under high pressure mixed with petrolatum ointment or Vaseline to be highly viscous [20].
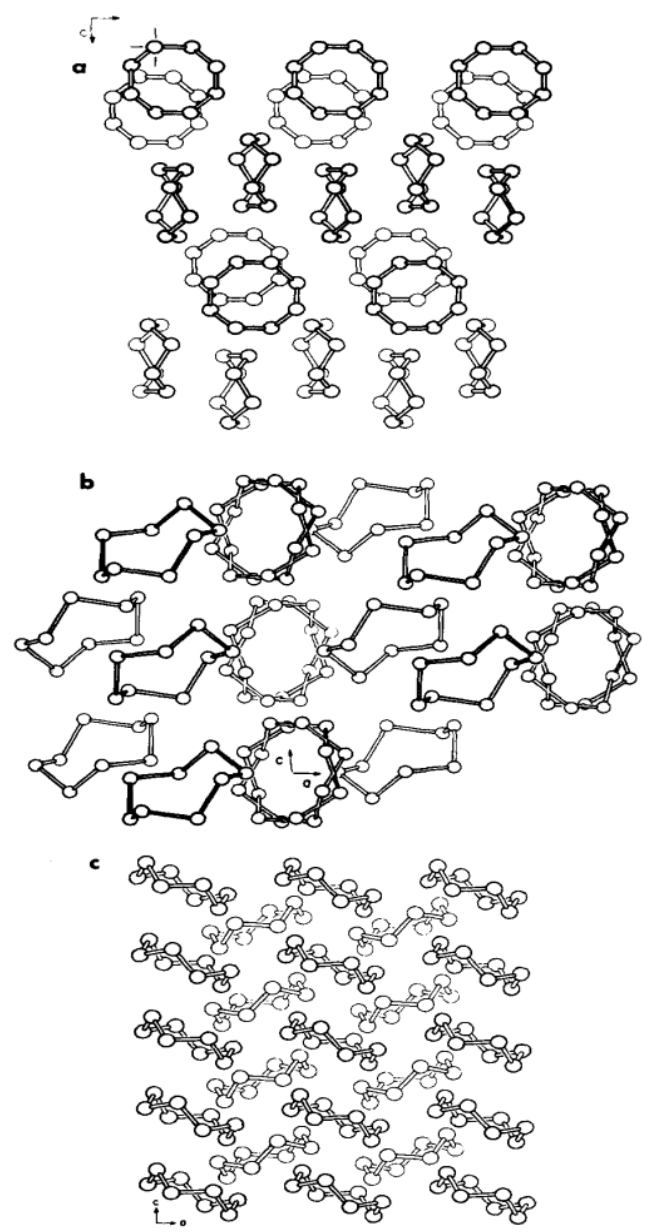

Figure1. The structure of the solid Figure 1 allotropes of Cycloocta $S:\left(\right.$ a) orthorhombic $S \alpha$, (b) monoclinic $S_{\beta}$, and (c) monoclinic $S_{\gamma}$. All views are perpendicular to the c axis [17].

\section{Materials and Method of Study}

Equation of state as a mathematical tool is used find relationship between fundamental properties of solid phase such as pressure and volume, without requirement of experimental methodology. Several equations of state have been developed to describe the behavior of solid materials under high pressure [22].

\subsection{Birch-Murnaghan EOS [23,24]}

$$
P_{\mathrm{B}-\mathrm{M}}=\frac{3 \mathrm{~B}_{0}}{2}\left(\eta^{-7 / 3}-\eta^{-5 / 3}\right)\left(1+\frac{3}{4}\left(\mathrm{~B}_{\mathrm{O}}^{\prime}-4\right)\left(\eta^{-2 / 3}-1\right)\right)
$$

Where, $\eta=\frac{V_{P}}{V_{0}}$

\subsection{Vinet EOS [25]}

$$
\begin{aligned}
& P_{\text {Vinet }}=3 B_{o}\left[\left(\frac{V_{\mathrm{P}}}{V_{o}}\right)^{-2 / 3}\left\{1-\left(\frac{V_{\mathrm{P}}}{V_{o}}\right)^{1 / 3}\right\}\right] \\
& \exp \left[\left(\frac{3}{2}\right)\left(B_{o}^{\prime}-1\right)\left\{1-\left(\frac{V_{\mathrm{P}}}{V_{o}}\right)^{1 / 3}\right\}\right]
\end{aligned}
$$

\subsection{Modified Lennard-Jones EOS [26]}

$P_{m L-J}=\frac{B_{o}}{n}\left(\frac{V_{o}}{V_{P}}\right)^{n}\left[\left(\frac{V_{o}}{V_{P}}\right)^{n}-1\right]$

Where $\mathrm{B}_{\mathrm{O}}$ : Isothermal bulk modulus at atmospheric pressure $B_{o}^{\prime}$ : First pressure Derivative of bulk modulus

Vo: Volume at ambient condition.

Vp: Volume under high pressure $\mathrm{P}$

$n=\frac{1}{3} B_{o}^{\prime} \quad ; \quad B_{o}^{\prime}=\frac{d B}{d p}$

\subsection{Bulk Modulus $(B)$}

The Bulk modulus $(B)$ relates the fraction change in volume to the change in the applied pressure as [27]:

$B=-V\left(\frac{d P}{d V}\right)_{T}$

On volume derivation of equations (1, 2, 3) and substituting in eq. (4) we can express the bulk modulus at pressure (p) as:

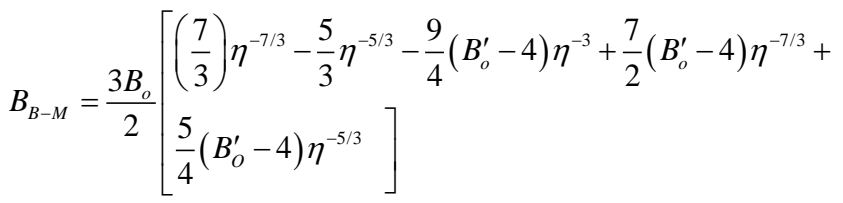

$B_{\text {Vinet }}=\left[2 B_{o}\left(\eta^{-2 / 3}-\eta^{-1 / 3}\right)+B_{o} \eta^{-1 / 3}+\frac{3}{2} B_{o}\left(B_{o}^{\prime}-1\right)\left(\eta^{-1 / 3}-1\right)\right]$

$\exp \left[\left\{\frac{3}{2}\left(B_{o}^{\prime}-1\right)\right\}\left(1-\eta^{1 / 3}\right)\right]$

$B_{m L-J}=B_{o}\left(\frac{V_{o}}{V}\right)^{n}\left[2\left(\frac{V_{o}}{V}\right)^{n}-1\right]$

\subsection{First Grüneisen Parameter $(\gamma)$}

The relation that describe $(\gamma)$ is given as $[28,29]$ :

$\gamma_{P}=\gamma_{0}\left(\frac{V_{P}}{V_{0}}\right)^{q}$

Where $\gamma_{0}$ : First Grüneisen parameter at atmosphere pressure. $\gamma_{P}$ : First Grüneisen parameter under high pressure $(\mathrm{P})$. 
$q$ : Second Grüneisen parameter.

$q$ Has been considered as equal to unity or a constant value [25].

\subsection{Debye Temperature $\left(\theta_{D}\right)$}

$\theta_{D}$ is a key parameter that determines the thermal transport dynamic properties. [30] Expressed Debye temperature under high pressure as:

$\theta_{D P}=\theta_{D o}\left(\frac{V_{o}}{V_{P}}\right)^{\gamma}$

$\theta_{D o}$ : Debye temperature at atmospheric pressure $\theta_{D P}:$ Debye temperature at pressure $(\mathrm{P})$.

\subsection{Phonon Frequency Spectrum (PFS)}

$v_{P}=v_{o}\left(\frac{V_{P}}{V_{o}}\right)^{-\gamma}$

The physical properties of solids can vary under high pressure, where the frequency of mode densities depend on specific volume eq. (10) [31]. The changes in the distribution function of the frequencies under high pressure expressed by eq. (11) [32].

$$
g\left(v_{P}, V_{P}\right)=\left(\frac{V_{P}}{V_{o}}\right)^{\gamma} g\left[v_{o}, V_{o}\right]
$$

$v_{o}:$ Frequency at atmospheric pressure

$$
v_{P}: \text { Frequency under high pressure }(\mathrm{P})
$$

\section{Calculations and Results}

\subsection{Compression Volume $\mathrm{V}_{\mathrm{P}} / \mathrm{V}_{0}$}

Table (1) values of bulk modulus $\left(B_{o}\right)$, its derivative $\left(B_{o}^{\prime}\right)$ and first Grüneisen parameter $\gamma$ for $S_{\beta}$ compared with $S \alpha$ taken from its original references.

\begin{tabular}{cccccc}
\hline Sulfur-type & $B_{o} \mathrm{Gpa}$ & $B_{o}^{\prime}$ & Ref. & $\gamma$ & Ref. \\
\hline $\mathrm{S} \alpha$ & 7.692 & 5.433 & {$[32]$} & 0.54 & {$[33]$} \\
$\mathbf{S}_{\boldsymbol{\beta}}$ & 7.046 & 5.597 & {$[32]$} & 0.54 & {$[33]$} \\
\hline
\end{tabular}

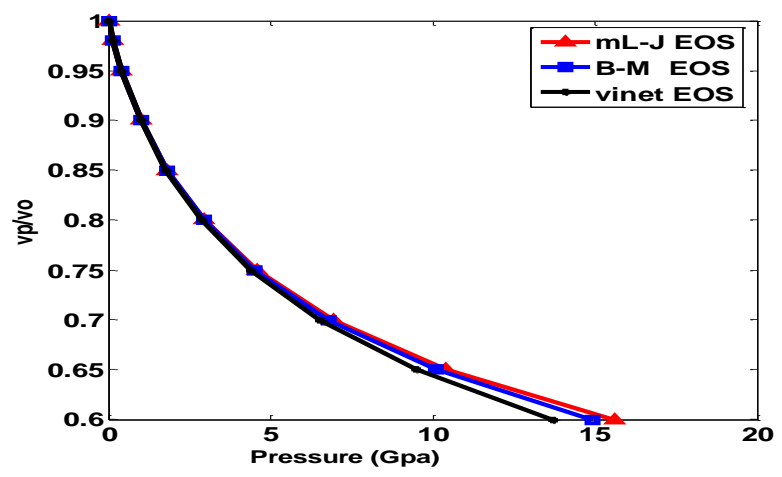

Figure 2. Variation of $V_{P} / V_{o}$ for $S_{\beta}$ at different values of pressure, using" $m L-J, B-M \&$ Vinet" EOSs.
By using data in Table (1) and using equations (1), (2) and (3) we get results for variations of $V_{P} / V_{0}$ with high pressure for $S_{\beta}$ which are shown in fig.(1).

\subsection{Bulk Modulus}

By substituting values of $B_{o}, B_{o}^{\prime}$ for $\mathrm{S}_{\beta}$ from Table (1) and $\mathrm{V}_{\mathrm{P}} / \mathrm{V}_{\mathrm{o}}$ rom Fig.(1) in equations (5),(6) and (7), we got the results of bulk modulus $\left(\mathrm{B}_{\mathrm{B}-\mathrm{M}}, \mathrm{B}_{\mathrm{mL}-\mathrm{J}}\right.$, and $\left.\mathrm{B}_{\text {Vinet }}\right)$ at different value of high pressure, and the results are shown in Fig. (3).

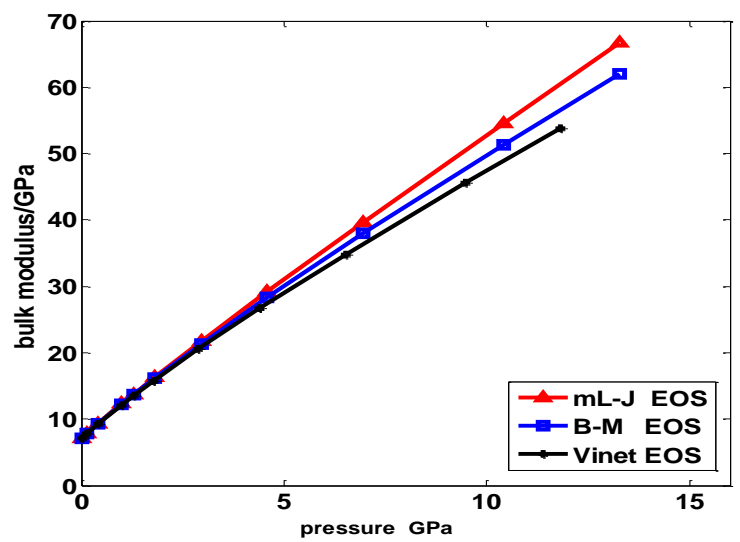

Figure 3. Variation of bulk modulus $B_{o}$ for $S_{\beta}$ at different values of pressure, using " $m L-J, B-M \&$ Vinet" EOSs.

\subsection{First Grüneisen Parameter}

First Grüneisen parameter $\gamma$ belongs to the most important physical characteristics of crystal lattice dynamics [34]. Where $\gamma$ is assumed to be volume dependent [35,36].

Fig.(4) show results for variation of $\left(\gamma_{P} / \gamma_{0}\right)$ for sulfur $\mathrm{S}_{\beta}$ under high pressure using data of $\mathrm{V}_{\mathrm{P}} / \mathrm{V}_{0}$ which is given from three equations (1), (2) and (3) at different value of pressure, and substituting it in eq.(8). Fig.4 shows a slightly reduction in Grüneisen parameter with increasing pressure from nearly 0.55 to just under 0.35 .

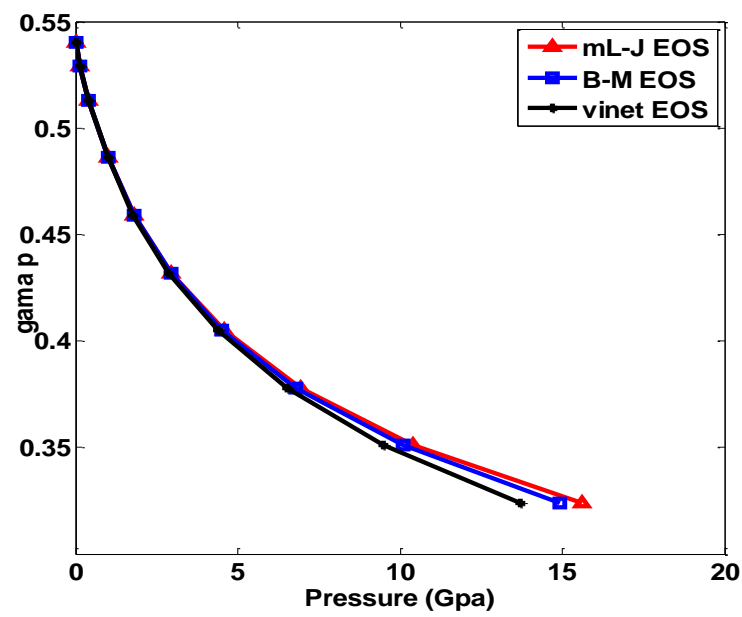

Figure 4. Variation of $\left(\gamma_{P} / \gamma_{0}\right) m L-J, B-M \&$ Vinet" EOSs.

\subsection{Debye's Temperature}

Figure (5) Shows results of variation of $\left(\theta_{D_{P}} / \theta_{D_{0}}\right)$ with high pressure for $S_{\beta}$ using equations (1), (2) and (3), when use $V_{P} / V_{0}$ values from Fig. (2) and substituting it in Eq. (9). 


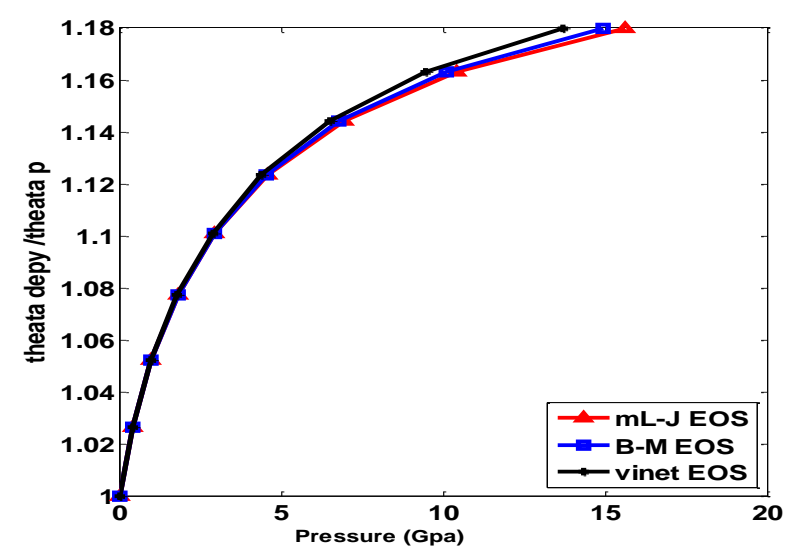

Figure 5. Variation of $\left(\theta_{D_{P}} / \theta_{D_{0}}\right)$ for $S_{\beta}$ at different values of pressure, using" m.L-J, B-M \& Vinet" EOSs.

\subsection{Phonon Frequency Spectrum (pfs)}

In the present work, the change in density of state (DOS) from (pfs) for sulfur is observed as expected by eq. (11). Fig. (6) show calculated pfs from literature [37]. by using values of $\mathrm{V}_{\mathrm{P}} / \mathrm{V}_{0}$ under high pressure from Fig.(1) and compiling in eq.(10) and eq.(11) for the three equations (1), (2) and (3) which give the results of pressure effect on pfs and illustrated in Figs (7, 8 and 9).

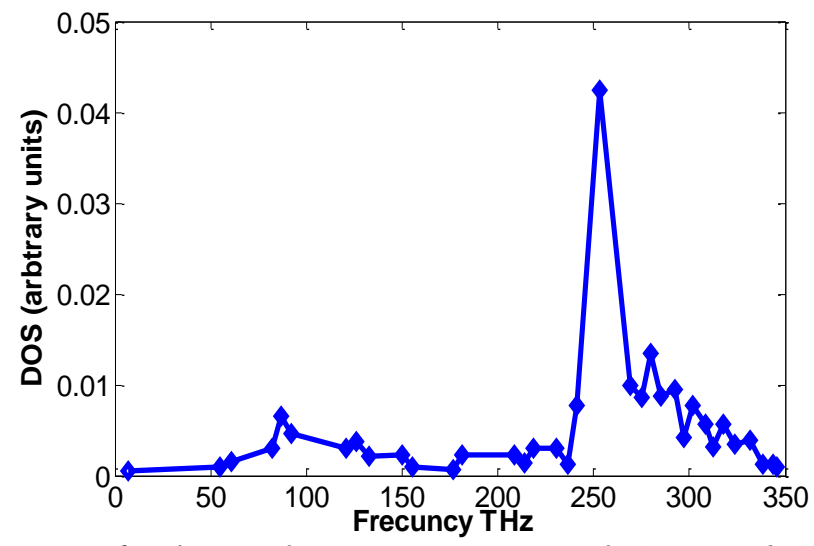

Figure 6. phonon frequency spectrum of $S_{\beta}$ at ambient condition [36].

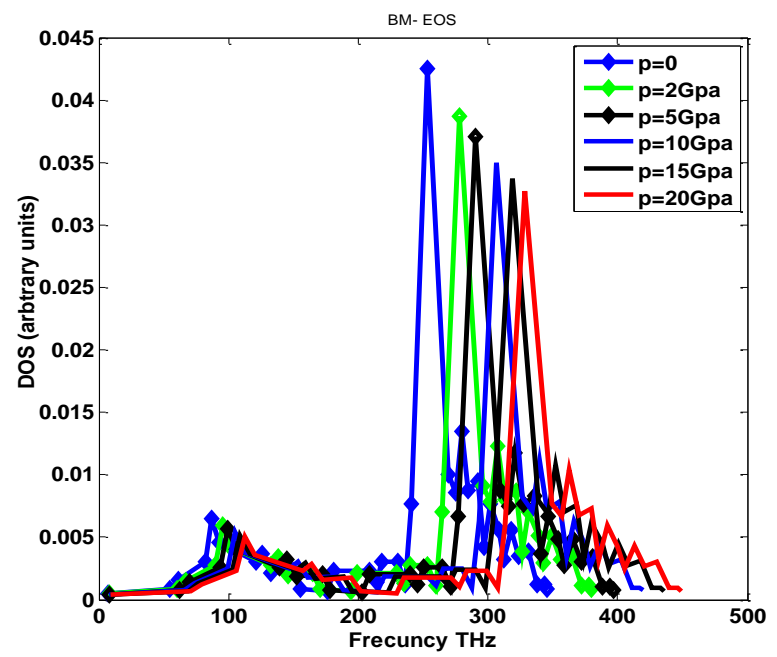

Figure 7. (pfs) for $S_{\beta}$ at different value of high pressure using (B-M EOS).

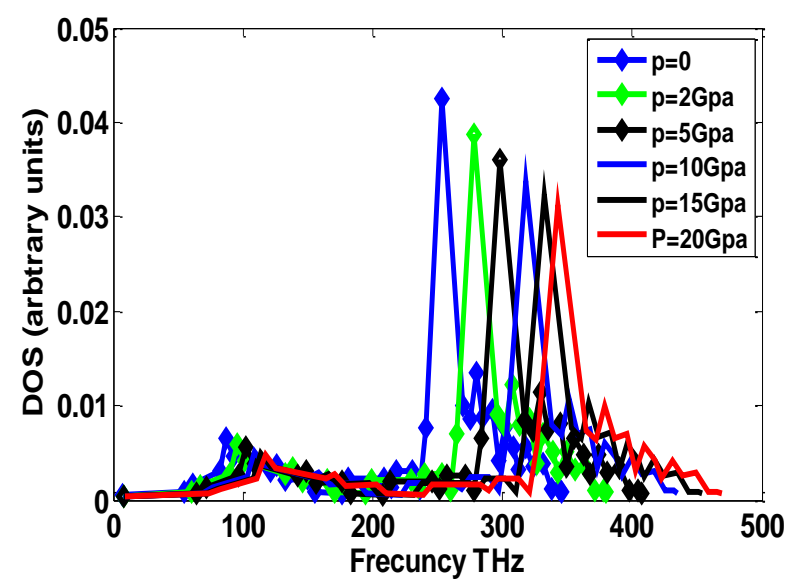

Figure 8. (pfs) for $S_{\beta}$ at different value of high pressure using (mL-J EOS).

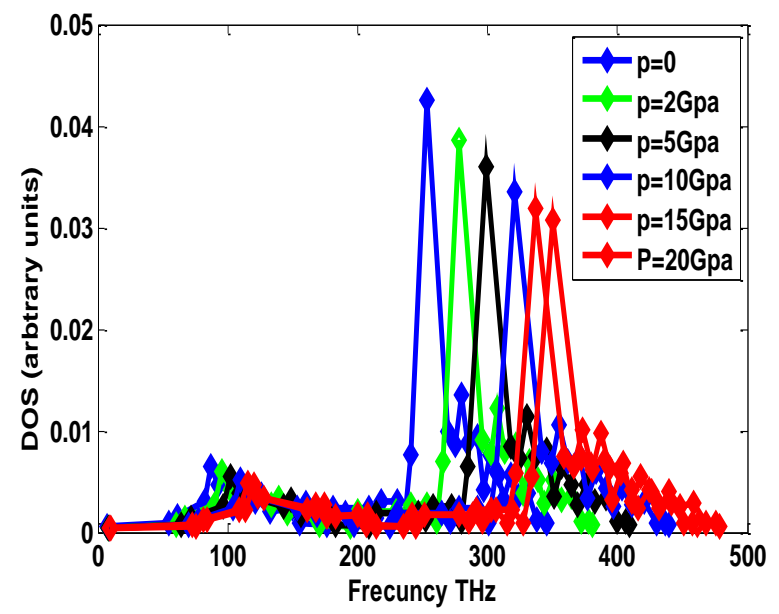

Figure 9. (pfs) for $S_{\beta}$ at different value of high pressure using (Vinet EOS).

\section{Results and Discussions}

The aim of this study is to develop a medicine uses to treat the germ of scabies, of which its size is $(0.25 \mathrm{~mm})$, and lives under the skin, we advise international pharmaceutical companies to use $\mathrm{S}_{\beta}$ treated under high pressure, less than "20Gpa" mixtures of Vaseline instead of $S_{\alpha}$, by comparing volume under high pressure for $S_{\beta}$ with germs size and grooves size that germs make, we found that $S_{\beta}$ molecules are able to get into the grooves through skin pores $(50 \mu \mathrm{m})$ and thus killing larvae when the eggs are hatching, unlike $S_{\alpha}$ that remains on the surface of the skin and does not enter the grooves because of its large size compared to $S_{\beta}$. To establish this purpose, the present work reviewed some important physical properties of sulfur $S_{\beta}$ have been studied under the effect of high pressure. Using three equations of state, its relative volume was observed under high pressure reaching 0.65 of its original size at pressures between $(9-11)$ GPa. Implementing the mathematical equations (1-11), the most important physical properties of sulfur were analyzed under high pressure up to $20 \mathrm{Gpa}$. The results were obtained and shown in Figures (1-8). The results in figs. 4-5 show an increasing trend for both bulk modulus and Debye temperature with increasing pressure. In fig. 5, it is noted that at lower pressure there is a more dramatic increase in Debye temperature, while as the pressure is increased more and more, the Debye temperature curve shifts up slowly, this can be attributed as a result of electrical repulsive force between adjacent atoms and molecules. On the other hand, the 
Grüneisen parameter and phonon density of state decreased with the application of high pressure. Regarding to the volume of $S_{\beta}$ particles, high pressure up to $16 \mathrm{Gpa}$ results in a dramatic change. Therefore, the present characteristics under strong compression results in only slight changes in other properties, with no change in chemical properties. All the studied properties gave excellent results for two equations (B-M EOS and $\mathrm{mL}-\mathrm{J}$ EOS) more than (Vinet EOS), but when we study the (pfs), equations (Vinet EOS and mL-J EOS) give better results than (B-M EOS).

\section{Conclusion}

High pressure effects on some vital thermo-elastic properties of sulfur $S_{\beta}$ material are analyzed such as relative volume, Grüneisen parameter, bulk modulus and phonon frequency spectrum. We have used some EOSs to derive formula for bulk modulus dependence on high pressure as given in Eqs. 5-7. Results in figs. 6-8 illustrate shifts in the energy of the modes (states) that high pressure causes exciting many new modes of vibration.

\section{Nomenclature}

$\begin{array}{ll}\mathrm{V}_{\mathrm{P}} / \mathrm{V}_{0} & \text { Volume compression ratio } \\ \mathrm{V}_{0} & \text { Volume at ambient condition } \\ \mathrm{V}_{\mathrm{P}} & \text { Volume under high pressure } \\ \text { EOS } & \text { Equation of state } \\ \mathrm{P}_{\mathrm{B}-\mathrm{M}} \text { EOS } & \text { Birch-Murnaghan equation of state } \\ \mathrm{P}_{\mathrm{mL}-\mathrm{J}} \text { EOS } & \text { Modified Lennard-Jones equation of state } \\ \gamma_{\mathrm{o}} & \text { First Grüneisen parameter } \\ \gamma_{\mathrm{P}} & \text { Grüneisen parameter under pressure } \\ B \mathrm{O} & \text { Bulk modulus } \\ v_{o} & \text { Frequency under ambient condition } \\ v_{P} & \text { Frequency under high pressure } \\ \theta_{D o} & \text { Debye temperature at atmospheric pressure } \\ \theta_{D P} & \text { Debye temperature under high pressure }\end{array}$

\section{Acknowledgments}

We thank university of Mosul, college of medicine, Nineveh Medicine College for helping in access to their laboratories. Thanks to Mr. Alaa Mohammad (New york Times journal correspondent), Ms. Faris M. Al hamadany (Msc. Phys., university of Newcastle, U.K., "work in Nineveh medicine collage") For reviewing the research. Thanks to "Directorate General of Education, Province of Nineveh/Iraq" for their moral and material support.

\section{References}

[1] S. K. Jalal, A. M. Al-Sheikh, R.H. Al-Saqa,"High Pressure Effects on the Phonon Frequency Spectrum of the Silicon Nanoparticale", Iran J. Sci. Technol. Trans Sci. 45, 391-396.2021 https://doi.org/10.1007/s40995$\underline{020-01041-9}$

[2] S. Rettig, J. Trotter," Refinement of the structure of orthorhombic sulfur, $\alpha-S 8$ ", Acta Crystallographica Section C: Crystal Structure Communications" vol. 43, (12), 2260-2262,1987.

[3] Y. Akahama, M. Kobayashi, H. Kawamura, "Pressureunduced structural phase transition in sulfur at $83 \mathrm{GPa} . "$, Phys. Rev. B. vol. 48, (10), 6862-6864. 1993.
[4] P. N. Gavryushkin, K. D.Litasov, S. S. Dobrosmislov, and Z. I. Popov," High-pressure phases of sulfur: Topological analysis and crystal structure prediction, Phys. Status Solidi B". 1600857.2017.

[5] A. V. Tobolsky and J. W. MacKnight, "Polymeric Sulfur and Related Polymers", Interscience, New York, N.Y., 1968.

[6] H. Luo, , R. G.Greene, A. L.Ruoff ,1993 "Beta-Po phase of sulfur at $162 \mathrm{GPa}$ : X-Ray diffraction study to 212 GPa." Physical Review Letters, vol. 71, (18), 29432946.1993.

[7] S. C. Abraham, "The crystal and molecular structure of orthorhombic sulfur," Acta Crystallogr. 8, 661.1955.

[8] A. Caron and J. Donohue, (1965), Acta Crystallogr. 18, 562.1995 .

[9] P. Coppens, Y. W. Yang, R. H. Blessing, Cooper W. F., Larsen, F. K.: J. Amer, Chem. Soc., Vol. 99, p760. 1977.

[10] R. W. Wyckoff, Crystal Structures, John Wiley \& Sons, New York.1963.

[11] M. Thackray, J. Chem. Eng. Data, vol. 15, 495. 1970.

[12] J. J. Trillat , J. Forestier, C. R., Acad. Sci., Paris, 192,(1931). 559. 1970.

[13] L. Gmelin's, "Handbuch der Anorganischen Chemie", Part 9A, 8th ed, Verlag Chemie. Weinheim. 1953.

[14] D. E., Sands, J. Am., Chem. Soc. 87, 1395,1965.

[15] L. M. Goldsmithand, and C. E. Strouse, "Molecular dynamics in the solid state. The order-disorder transition of monoclinic sulfur", J. Am. Chem. Sot., 99, 75807589,1977 .

[16] Y. Watanabe, Acta Crystallogr., Sect. B,30. 1396,1974.

[17] Y. M. de Haan, Physica, 24, 855,1958

[18] E. Meyer. E d.,"Elemental Sulfur, Chemistry and Physics", Inter- science, New York, N.Y,1965.

[19] N. Ishii, "Guideline for the diagnosis and treatment of scabies in Japan" , (second edition). Journal of Dermatology 35, 378-393,2008.

[20] k. Sharquie, J. Al-Rawi, A. Noaimi and H.Al-Hassany, "Treatment of scabies using $8 \%$ and $10 \%$ topical sulfur ointment in defferent regimens of application", J Drugs Dermotol., 11, Mar, (13):375-64,2012.

[21] H. Luo, A. L. , "Ruoff, X-ray-diffraction study of sulfur to $32 \mathrm{GPa}$ : Amorphization at 25GPa", Phys. Rev. B, 48, (1), 569.1993.

[22] R. H. AL-Saqa, S. J. AL-Taie,"Theoretical Study of Mechanical, Elastic and Phonon Frequency Spectrum Properties for GaAs at High Pressure" , Journal of Siberian Federal University. Mathematics \& Physics, 12, (3), 371-378,2019.

[23] F. Birch," Finite elastic strain of cubic crystal", Phys. Rev. Vol. 71, 809- 824,1947.

[24] A. M. Al sheikh, S. K. Jalal, S. A. Mawlood, "Theoretical High Pressure Study of Phonon Density of State and Debye Temperature of Solid C60: Grüneisen Approximation Approach", International journal of 
thermodynamics, vol. 25, No. 1, 2022. https://doi.org/10.5541/ijot.900071.

[25] P. Vinet, J. Ferrante, J. Rose, and J. Smith, " Compressibility of solids", J. .Geophys. Res., vol. 92, 9319-9325,1987a.

[26] S. Jiuxun, "A modified Lennard-Jones type equation of state for solids strictly satisfying the spinodal condition", J. Phys.: Condens. Matter, vol. 17, L103-L111,2005.

[27] R. Boehler, "Melting temperature, diabetes and Gruneisen parameter lithium, sodium and potassium versus pressure", Phys. Rev. B., 127, No. 11, pp. 6754$6762,1993$.

[28] A. M. Al-sheikh, S. K. Jalal, R. H. Al-saqa, "Equation of State and Thermo Dynamic Behaviour of C60 under High Pressure", Universal Journal of Mechanical Engineering 8, (1), 59-65,2020. DOI: 10.13189/ujme.2020.080108

[29] A. M. Al-Sheikh, S. J. Al-Faris, S. K. Jalal, " Grüneisen Parameter Variation Consideration in Theoretical High Pressure Studies for C60", Iran J. Sci. Technol. Trans Sci. A, 46, 2022. https://doi.org/10.1007/s40995-02101253-7.

[30] R.S. Preston, S.S. Hanna, J. Helerle(1962), Mössbaur effect in metallic Iron. Phys. Rev., 128, (5), 2207-2218.

[31] J. Dlouha., " The influence of pressure on the Mössbauer effect", Czech. J. Phys., B, vol. 14, 571-579,1964.
[32] P.W. Guan a, S.-Li Shang, G. Lindwall, T. Anderson b, Z.-Kui Liu,"First-principles calculations and thermodynamic modeling of the $\mathrm{S}$-Se system and implications for chalcogenide alloys", Journal of Alloys and Compounds, vol. 694, 510-521,2017.

[33] G. A. Saunders, Y. K. Yoḡurtçu , J. E., "Macdonald and Godfrey Stuart Pawley, The elastic behavior of orthorhombic sulfur under pressure", The Royal Socilty Open Scince Journal, ,vol. 407. Issue 1883,1986.

[34] Q. Lio, L. R. Chen., "Pressure dependence of the Grüneisen parameter and thermal expansion c oefficient of solids" , Indian Journal of pure \&applied physics, vol. $55,368-371,2017$

[35] S. Kareem Jalal, A. M. AL-Sheikh, "Theoretical High Pressure Study for Thermoelastic Properties of NaClB1", Rafidain Journal of Science, Volume 25, Issue 6, Pages 80-89,2014. Doi 10.33899/rjs.2014.131357.

[36] S. J. AL-Faris , R. H. Al-Saqa, H. M. Mohammed and S. K. Jalal, "High pressure effects on the structural properties of GaN compound using equations of state ", International journal of thermodynamics, vol. 25, No. 1,2022. https://doi.org/10.5541/ijot.960849.

[37] M. Güler, E. Güler, "Theoretical Analysis of Elastic, Mechanical and Phonon Properties of Wurtzite Zinc Sulfide under Pressure", Crystals, 7, 161,2017. 